Primljen/Received: 29. 3. 2021. Prihvaćen/Accepted: 10. 5. 2021.
UDK/UDC 821.112.2.09-32Hoffmann E. T. A.:75

Pregledni znanstveni članak/Scientific review article

\title{
Anja Orozović
}

\section{FUNKTIONEN DER BILDENDEN KUNST IM LITERARISCHEN WERK E. T. A. HOFFMANNS}

E. T. A. Hoffmann war ein vielfach begabter Künstler, der sich zwar primär als Schriftsteller, aber auch als Komponist und Zeichner betätigte. Diese Begabungen lassen sich anhand verschiedener Spuren innerhalb seines literarischen Euvres verfolgen. Die Bezüge zur bildenden Kunst prägen Hoffmanns Gesamtwerk von der Früh- bis zur Spätphase. Seinem Interesse an bestimmten Künstlern oder Werken, die Hoffmann sowohl in der Dresdener Gemäldegalerie als auch während der Bamberger und Berliner Periode rezipierte, begegnet man in Form verschiedener Verweise vor allem innerhalb seiner Erzählungen. Die vorliegende Darstellung umfasst die Erzählsammlungen Fantasiestücke in Callots Manier. Blätter aus dem Tagebuche eines reisenden Enthusiasten (1814/15), Nachtstücke (1816/17) sowie Die Serapionsbrüder (1819/21). Dabei wird einleitend auf Hoffmanns Auseinandersetzung mit verschiedenen künstlerischen Vorbildern eingegangen, um anschließend die Spuren dieser fruchtbaren Begegnungen zu beleuchten. Der Beitrag teilt die Verweise in vier Kategorien ein, die innerhalb ausgewählter Erzählungen verschiedene Funktionen aufweisen. Hoffmann stützt sich zum einen auf die traditionell malerischen Gattungen der Fantasie-und Nachtstücke und versucht deren Merkmale in die Literatur zu transponieren. Darüber hinaus dienen die Werke bildender Künstler Hoffmann ebenfalls als Erzählanlass bzw. prägen seine Protagonisten- und Landschaftsdarstellungen. Die Analyse dieser intermedialen Verbindungen soll zum besseren Verständnis von Hoffmanns Erzählwerk sowohl auf der poetologischen als auch der inhaltlichen Ebene beitragen.

Schlüsselbegriffe: E. T. A. Hoffmann, Romantik, Intermedialität, Literatur, Malerei

\section{EINFÜHRUNG}

Das literarische Werk E. T. A. Hoffmanns wurde durch seine musikalischen, zeichnerischen und malerischen Begabungen geprägt und erweist sich als repräsentativ für die erstrebte Kunstsynthese der romantischen 
Literatur. Man kann sagen, dass ,[es] zur besonderen Existenz Hoffmanns gehört, dass er ein Kernstück der romantischen ästhetischen Theorie $z u$ leben versuchte.“ (Göbel, 1992, S. 149) Neben seinen Erfolgen als Schriftsteller und Komponist betätigte sich Hoffmann ebenfalls als Karikaturist und Maler. Seine Karikaturenzeichnungen bezeugen sowohl das reiche Talent als auch die blühende Fantasie des Künstlers, jedoch traten Hoffmanns malerische Ambitionen im Verlauf seiner künstlerischen Entwicklung in den Hintergrund und fanden trotz der Bemühungen wenig Anklang. (Vgl. Ponert, 2012a/ 2012b; Göbel, 1992, S. 149- 165; Böttcher, 1980) Die Abkehr von der Malerei versuchte Hoffmann literarisch zu kompensieren. Sein literarisches Werk wurde durch die Auseinandersetzung mit zahlreichen bildenden Künstlern beeinflusst, denen man durch explizite Verweise vor allem innerhalb seiner Erzählungen wiederbegegnet. Wie Gerhard Kaiser (1988, S. 145) betont, ,[ist] Hoffmanns sprachliche Bildlichkeit aber auch dort noch stark bildkünstlerisch bestimmt, wo ein Bezug nicht ausdrücklich im Text hergestellt wird.“ Die eindeutigen Bezüge enthalten die Titel der Erzählsammlungen wie Fantasiestücke in Callots Manier. Blätter aus dem Tagebuche eines reisenden Enthusiasten (1814/15) oder Nachtstücke (1816/17). Zahlreiche Referenzen können ebenfalls in der Sammlung Die Serapionsbrüder (1819/21) oder auch im Spätwerk Prinzessin Brambilla. Ein Capriccio nach Jakob Callot (1820) vorgefunden werden. Der vorliegende Beitrag schließt aufgrund des Umfangs das Spätwerk aus.

\section{E. T. A. HOFFMANNS KÜNSTLERISCHE VORBILDER}

Unterschiedliche malerische Vorbilder erweisen sich durch verschiedene Entwicklungsphasen der dichterischen Laufbahn als entscheidend für die Bestimmung und Begründung von Hoffmanns Poetik. (Vgl. Braun, 2004; Wittkop-Ménardeu, 1996; Kleßmann 1988; Safranski, 1984) Eine Beschäftigung mit dem englischen Maler und Zeichner William Hogarth (1697-1764) weist schon das Frühwerk auf. Hogarths Gemälde und Zeichnungen charakterisieren satirische Darstellungen des bürgerlichen Lebens, welche unter anderem auch die Position und Rolle des Künstlers innerhalb der Gesellschaft - ein beliebtes Thema Hoffmanns - ansprechen. Hartmut Steinecke (2004, S. 61 ff.), sowie auch Detlef Kremer (2009, S. 88 f.) betonen, dass der Bezug zu Hogarth sowohl die Erzählungen der Kreisleriana 
(1814/15) als auch allgemein die Sammlung der Fantasiestücke prägt, welche ursprünglich den Titel Bilder nach Hogarth tragen sollte.

Als Hoffmanns wichtigstes künstlerisches Vorbild erweist sich jedoch der französische Kupferstecher Jaques Callot (1592 - 1635). Im ersten Teil der Fantasiestücke äußert sich Hoffmann einleitend über sein (künstlerisches) Idol und bezeichnet Callot begeistert als den „kecke[n] Meister“ (Hoffmann: 1963a, 62) der ,sonderbaren phantastischen Blätter[].“(ebd.) Er lobt die „überreichen, aus den heterogensten Elementen geschaffenen Kompositionen“ (ebd.), in denen Callot die ,phantastischen wunderlichen Erscheinungen, die der Zauber seiner überregen Phantasie hervorrief" (ebd.) in einem fantastischen Wirbel aufeinanderstoßen lässt. Detlef Kremer (1998, 40) erklärt dabei, dass Hoffmanns Äußerungen über Callot „,weniger ein kunstgeschichtlicher Exkurs als eine Art poetologischer Selbstkommentar" sind, da ,[Hoffmann] in den Stichen Callots eine Spiegelung seiner Poesie des »Phantastischen« [sieht].“ (ebd.) Durch die „die Figurenhäufungen und -verwandlungen“ (ebd.) der Fantasiestücke lässt Hoffmann fantastische Welten entstehen, wo ,sich das Alltägliche mit dem Wunder vermischt, die Grenzen zwischen Mensch und Tier, Ironie und Ernst in Fluss geraten und etwas »fremdartig Bekanntes« (II/ 1 17) entsteht, dessen Paradoxie dafür Gewähr zu leisten hat, dass es nicht auf Begriffe des Verstandes zu reduzieren ist.“ (ebd.) Sein Kunstziel erweist sich daher als ,jene fantastische Überformung der Realität, durch die ihm auch die Anknüpfung an das Schaffen des französischen Kupferstechers naheliegt.“ (Schnyder, 1998, S.14)

Eine Vielfalt an fantastischen Welten, wo „das Unwahrscheinliche, Unerwartete, Unangemessene, ja Unmögliche aufeinanderstoßen“ (Steinecke, 2004, S. 89.), findet Hoffmann nicht nur bei Hogarth oder Callot. Die fantastischen Bilder von Hieronymus Bosch (um 1452-1516) oder Jan Brueghel dem Älteren (1586-1625) entsprechen ebenfalls diesem Anspruch, vor allem weil sie in der kunsthistorischen Rezeption der Gattung des Nachtstücks zugeordnet werden. Der unerwartete und bedrohliche Zusammenstoß zweier Welten, der die Werke Callots, Boschs oder Brueghels kennzeichnet, gilt ebenfalls als Charakteristikum von Hoffmanns Poetik. In den anscheinend ordinären Alltag seiner Protagonisten brechen fantastische Ereignisse, seltsame und schauerliche Gestalten ein. Die Grenze zwischen der Realität und Fantasiewelt, welche vielleicht nie existierte, wird 
unerwartet aufgelöst und die Protagonisten in ein Reich des Wunderbaren und Geheimnisvollen gelockt, aus dem es meistens keine Fluchtmöglichkeit gibt.

Die erwähnte Vorliebe für das Schauerliche und Bizarre äußert sich ebenfalls in Hoffmanns Bezug zu Salvator Rosa (1615-1673), dessen Landschaftsdarstellungen durch intensive Licht-Schatten-Kontraste die Naturgewalt bis an die Grenze des Apokalyptischen steigern. Verschiedene Referenzen auf Rosa sind besonders in der Sammlung Die Serapionsbrüder zu finden. Neben den bereits genannten Künstlern muss ebenfalls Hoffmanns Interesse für Albrecht Dürer (1471-1528), Rembrandt (1606-1669), Anthonis van Dyck (1599-1641) ${ }^{1}$ und Frans van Mieris (1635-1681) erwähnt werden. Seine Auseinandersetzungen mit diversen künstlerischen Vorbildern weisen innerhalb der Erzählungen verschiedene Funktionen auf, welche folgend überblicksweise präsentiert werden.

\section{FANTASIE- UND NACHTSTÜCKE: LITERARISIERUNG DES FANTASTISCHEN UND SCHAUERLICHEN}

Wie Steinecke (2004, S. 157) in der Erläuterung zu den Fantasiestücken betont, „[ist] »Fantasie« der Schlüsselbegriff des Werkes und einer der zentralen Begriffe zur Kennzeichnung von Hoffmanns Gesamtwerk.“ Die Literarisierung fantastischer Vorgänge und Erscheinungen erwies sich für Hoffmann als Möglichkeit, Literatur und Malerei miteinander zu verschmelzen, da sich beide Künste bei der Gestaltung des Fantastischen mit unterschiedlichen Darstellungsmitteln bedienen. Hoffmann übernimmt nicht nur die ursprünglich malerischen Gattungen der Fantasie- und Nachtstücke für seine Erzählsammlungen. Er versucht zugleich die fantastischen und schauerlichen Verwandlungsprozesse durch Verweise auf malerische Licht-Schatten-Effekte oder auch konkrete Künstler literarisch zu suggerieren. Die Bezüge zur Malerei weisen somit im Rahmen der Fantasieund Nachtstücke eine Doppelfunktion auf.

\footnotetext{
${ }^{1}$ Verschiedene Verweise auf die genannten Künstler lassen sich innerhalb der Erzählungen Meister Martin der Küfner und seine Gesellen (1818) und Der Artushof (1816) vorfinden, worauf aufgrund des Umfangs dieser Arbeit nicht detaillierter eingegangen werden kann.
} 
In diesem Zusammenhang betont Stephan Reher (Vgl. 1997, S. 29-33), dass sich das Fantastische gerade durch den Verwandlungsprozess offenbart, der als ein zeitlicher Vorgang primär dem Darstellungsvermögen der Literatur, sekundär der Malerei zugesprochen wird. Trotzdem suchten die Autoren in den Werken der Malerei nach der Quelle des Fantastischen. Gerade Boschs, Breughels oder Callots überfüllte, groteske und fantastische Szenen schaffen es, durch die Darstellung mehrerer gleichzeitig ablaufender Aktionen Verwandlungsprozesse zu suggerieren. Die Vorliebe für fantastische Begebenheiten und Gestalten, einschließlich des Interesses an den erwähnten Künstlern übernimmt auch Hoffmann. Die expliziten Titelbezüge der Fantasie- und Nachtstücke bestätigen somit das intermediale Anliegen seiner Poetik.

Die Titel beider Erzählsammlungen enthalten „das Bestimmungswort »-stück«, [das - Anm. d. Verf.] ,seit dem 16. Jahrhundert ein künstlerisches Werk [bezeichnet], insbesondere in der bildenden Kunst und der Musik, dann auch in der Literatur (besonders angewandt auf die Bühnendichtung.)“ (Steinecke, 2004, S. 158) Dabei soll betont werden, dass Hoffmann die Bezeichnung »Stück« primär im Kontext der Malerei verwendet.“ (ebd f.) Die Erzählungen der Fantasiestücke, genauer „,»fantastische Gemälde in der Art Callots«“" (ebd.), erweisen sich daher als ,heitere fantastische Bild-Text-Metamorphosen, ganz so wie die »Nachtstücke» Transformationen düsterer, unheimlicher Bilder sind.“ (Kremer/Klicher 2015, S. 172) Hoffmann gelingt somit eine ,ungewöhnliche Bedeutungserweiterung“ (Steinecke, 2004, S 158 f.) des Begriffs, indem er „die drei zentralen Künste [verschmilzt]“ (ebd.) und somit „ein programmatisches Beispiel seines Strebens nach einer Universalkunst“ (ebd., S. 159) vorlegt. Die ursprünglich malerischen und musikalischen Gattungen wurden somit in den Bereich der Literatur transponiert.

Hoffmanns Absicht war es, sowohl fantastische als auch schauerliche Erscheinungen literarisch darzustellen, indem er die spezifischen Darstellungsmöglichkeiten der Literatur und Malerei verbindet. So verknüpft er das Vermögen der Literatur, „fantastische Handlung, die Verwandlung des Bekannten in das Furchterregende, den plötzlichen Umschwung [beizusteuern]“, (Reher, 1997, S. 33) mit dem Vermögen der Malerei „eine reine Atmosphäre, eine nicht recht erklärbare Stimmung von Unwirklichkeit [aufzubauen].“ (ebd.) Derartiges versucht Hoffmann innerhalb seiner 
bekannten Erzählung Der goldene Topf (1814) zu erreichen, wo die ,angesiedelte[] bürgerliche[] Alltagswelt und eine[] Ebene des märchenhaft Wunderbaren“ (Schmaus, 1998, S. 30) aufeinandertreffen. Das Fantastische, zugleich aber auch Schauervolle dieser Erzählung offenbart sich durch die zahlreichen Verwandlungsszenen. Als eine der einprägsamsten erweist sich die Szene der zweiten Vigile, wo sich der bronzene Türklopfer in das Gesicht des alten Apfelweibs transformiert. Einen expliziten Bezug zu den malerischen »Fantasiestücken« weist jedoch die zentrale Äquinoktialnacht-Szene der siebten Vigile auf, in der Veronika mit Hilfe der alten Rauerin versucht, den Geliebten Anselmas aus der Zauberwelt des Archivarius Lindhorst zu befreien. Aus dem ,blitzenden roten Strahlen und Funken“ (Hoffmann, 1963a, S. 330f.) der ,emporflackernden Flammen“ (ebd.) entsteht ein Wirbel von Licht und Bewegung, aus dem sich das vom „Entsetzen“ (ebd.) verzogene Gesicht des Mädchens hervorhebt und ihr Körper gleichzeitig zu einem „Marmorbild“ (ebd.) erstarrt. Damit endet der Schrecken aber nicht. Durch die Erscheinung auf dem Kreuzweg wird noch die Gestalt der alten Hexe, ,ein langes, hageres, kupfergelbes Weib mit spitzer Habichtsnase und funkelnden Katzenaugen“ (ebd.) herbeigeführt.

Reher (1997, S. 65) betont, dass Hoffmann die Äquinoktialnacht-Szene „komplett mit Lichtverhältnissen, Farben und Formen, aber fast ohne Geräusche, sogar ohne Lautmalerei, ohne wörtliche Rede, ohne Veränderung“ literarisch ausmalt. Mit der Darstellung, die als ,eine Art Geistertanz mit schaurigen Gestalten und einer angsteinflößenden Hexe“" (ebd.) konzipiert wurde, erschafft Hoffmann ,in der Tat eine Art Bilderbeschreibung ohne zugrundeliegendes Original, ein Gemälde aus Worten.“ (ebd.) Die Referenz auf „Rembrandt und Höllenbreughel“" (Hoffmann, 1963, S. 327) dient dabei ,als Ergänzung des Gesamteindrucks, sozusagen als Hinweis auf den malerischen Stil, in dem das imaginäre Bild gehalten ist.“ (Reher, 1997, S. 65) Dabei wird erwähnt, dass ein solches Motiv eher Breughel als Rembrandt zugeschrieben werden kann, obwohl auch Breughels Darstellung ,typischerweise als Teil einer großen Vision bei Tageslicht erscheinen [würde - Anm. d. Verf.], um die gezeigten Fantasiegestalten optimal zur Geltung zu bringen.“ (ebd.) Hoffmanns Absicht synthetisiert die stilistischen und motivischen Besonderheiten sowie die Gestaltungsmittel beider Maler. „Von Rembrandt nimmt Hoffmann also die Beleuchtung mit einem 
Breughel-Motiv und schafft somit ein Kunstwerk aus Worten, dessen UrBild ein ,Gemeinschafts-Gemälde" von Brueghel und Rembrandt wäre.“ (ebd., S. 66) Hoffmann lässt somit „,ein fantastisches Nachtstück““ (ebd.) entstehen, das die Szene mit einer Stimmung des Entsetzens erfüllt.

Bei der später veröffentlichten Erzählsammlung der Nachtstücke geht Hoffmann ähnlich vor. Die Gattung des Nachtstücks entstammt ebenfalls aus der Malerei. Die ursprünglichen Bezeichnungen »quadro di note« oder "pittura di notte« stammen von Giorgio Vasari und beziehen sich auf malerische Nachtszenen des 15. Jahrhunderts. (vgl. Steinecke, 2004, S. 260f.) Reher (vgl. 1997, S. 38f.) stützt sich bei seiner Behandlung dieses Themas auf kunstgeschichtliche Deutungen, die das Nachtstück als eine in nächtlicher Erleuchtung erscheinende malerische Darstellung auffassen. Die Bezeichnung Nachtstück wurde primär im Kontext der Malerei geläufig, die sich allgemein der Schauer, Nacht- und Mordmotivik zuwendet. Schon im 17. Jahrhundert wurde die Bezeichnung in die Literatur, genauer in den Bereich der populären Schauerliteratur übertragen. Steinecke (2004, S. 261) betont, dass Hoffmanns Rezeption auf ,eine relativ geläufige und im populären Kontext stehende Bezeichnung [zurückgreift], auch wenn der Begriff immer noch in erster Linie auf die bildende Kunst angewandt wurde und mehr eine Darstellungstechnik meinte als eine literarische Gattung." Obwohl Hoffmanns Auffassung des Nachtstücks die zeitgenössische Schauerliteratur-Mode verfolgt, lösen sich die Bezüge zur Malerei innerhalb seiner Erzählungen nicht auf. Sowohl das malerische als auch das literarische Nachtstück zeichnet sich durch ein bestimmtes Motivspektrum, genauer ein ,übernatürliches beziehungsweise in übernatürlicher Weise dargestelltes Motiv“ (Reher, 1997, S. 74) aus. Dazu wird das Nachtstück durch das „Lichtverhältnis [bestimmt - Anm. d. Verf.], in dem sich das dargestellte Motiv befindet. Und die Darstellung dieses Lichtverhältnisses wiederum ist die Domäne der Malerei.“ (ebd., S. 33) Hoffmann versucht daher die Schauerstimmung seiner Nachtstücke sowohl durch eine bestimmte Motivauswahl als auch die Darstellung der Hell-Dunkel-Effekte zu erreichen. Dabei wirkt das optisch Wahrgenommene auf das Innere der Protagonisten, indem es oft ,zum Einfallstor für manipulative Beeinflussung oder affektive Selbsttäuschung“ (Schneider, 2015, S. 56) wird.

Die Hexenszene aus den Erlebnissen des Hundes Berganza (1814) fasst Reher als ein solches übernatürliches Motiv auf. Die Erzählung gehört 
nicht zu der Sammlung der Nachtstücke, aber hier verbindet Hoffmann gerade die fantastische Verwandlung mit dem übernatürlichen Motiv, das in einer nächtlichen Erleuchtung dargestellt wird. Im Mittelpunkt der Erscheinung steht „ein seltsam geformter Kessel“ (Hoffmann, 1963a, S. 156 f.), bei dem eine ,in häßlichen glänzenden Farben gesprenkelte Kröte saß“ (ebd.) und „,mit einem langen Löffel darin [rührte], daß schäumend, zischend und prasselnd der kochende Gischt übergärte in die Flammen hinein, aus denen blutrote Funken emporfuhren, die in garstigen Gebilden zur Erde fielen.“ (ebd.) Um sie herum sammeln sich „Eidechsen mit albern lachenden Menschengesichtern, spiegelglatte Iltisse, Mäuse mit Rabenköpfen, allerlei widriges Ungeziefer.“ (ebd.) In der Szene wimmelt und krabbelt es von schauerlichen Gestalten, die aus der nächtlichen Dunkelheit herauskriechen, um wieder darin zu verschwinden, sodass das farbige Licht des Hexenkessels die Wahrnehmung des Beobachters verwirrt. Bei dieser Szene sieht Reher einen expliziten Bezug zu Hieronymus Bosch beziehungsweise zu dem Motiv des „Hexensabbat[s] mit Tieren, die Teufelsattribute sind und Mischwesen aus Mensch und Tier“ (Reher, 1997, S. 74) darstellen. Dagegen betont Steinecke (vgl. 2004, S. 160), dass bei der Hexenabbat-Szene ein direkter Bezug zu Callot besteht, den Hoffmann auch in einem Brief an seinen Verleger Kunz betont. Somit werden erneut motivische und stilistische Besonderheiten zweier Maler zu einem literarischen Gemälde verschmolzen, das von einer Schauerstimmung erfüllt werden soll.

Bei der Gestaltung der literarischen Fantasie- und Nachtstücke geht Hoffmann ähnlich vor. So lässt sich bei den vorliegenden Beispielen auch keine klare Trennung zwischen einem Fantasiestück und einem Nachtstück vollziehen, da Hoffmann innerhalb ausgewählter Szenen die motivischen und stilistischen Elemente beider Gattungen verbindet. Er übernimmt erstmals ein für beide Gattungen spezifisches Motiv wie z. B. das Zauberritual einer Hexe. Dieses Motiv wird dann als ein fantastischer Verwandlungsprozess in nächtlicher Beleuchtung dargestellt, um schließlich durch die Verweise auf malerische Gegenstücke die Bildlichkeit der jeweiligen Szene zu steigern. Dabei geht es Hoffmann nicht um eine Art Nachahmung oder einfache Identifikation mit den Werken bestimmter Maler. Die von Hoffmann entworfenen „,Gemeinschafts-Gemälde““ (Reher, S. 66) erweisen sich als eigene spielerische Interpretation der Fantasie-und Nachtästhetik. 


\section{DAS GEMÄLDE ALS ERZÄHLANLASS}

Zahlreiche Referenzen auf bildende Künstler oder konkrete Gemälde kommen auch innerhalb der späteren Erzählsammlung Die Serapionsbrüder vor und weisen dabei verschiedene Funktionen auf. Die erste Funktion ist daran erkennbar, dass bei einigen Erzählungen ,die Betrachtung und Beschreibung von konkreten, historisch nachweisbaren Gemälden als Auslöser des Erzählens fungieren [kann - Anm. d. Verf.].“ (Risi, 1998, S. 359) In diesem Zusammenhang sei erstmals auf die Erzählung Die Fermate (1816) verwiesen. Als Bildvorlage diente Hoffmann Johann Erdmann Hummels Gemälde Gesellschaft in einer italienischen Lokanda, das 1814 auf der Berliner Kunstausstellung präsentiert wurde. Als eigentlicher Gegenstand dieser Erzählung, die mit einer Gemäldeschilderung beginnt, erweist sich jedoch die Musik.

- Eine üppig verwachsene Laube - ein mit Wein und Früchten besetzter Tisch - an demselben zwei italienische Frauen einander gegenübersitzend - die eine singt, die andere spielt Chitarra - zwischen beiden hinterwärts stehend ein Abbate, der den Musikdirektor macht. Mit aufgehobener Battutapaßt er auf den Moment, wenn Signora die Kadenz, in der sie mit himmelwärts gerichtetem Blick begriffen, endigen wird im langen Trillo, dann schlägt er nieder und die Chitarristin greift keck den Dominanten-Akkord. - Der Abbate ist voll Bewunderung - voll seligen Genusses - und dabei ängstlich gespannt. - Nicht um der Welt willen möchte er den richtigen Niederschlag verpassen. Kaum wagt er zu atmen. Jedem Bienchen, jedem Mücklein möchte er Maul und Flügel verbinden, damit nichts sumse. Um so mehr ist ihm der geschäftige Wirt fatal, der den bestellten Wein gerade jetzt im wichtigsten höchsten Moment herbeiträgt. - Aussicht in einen Laubgang, den glänzende Streiflichter durchbrechen. - Dort hält ein Reiter, aus der Lokanda wird ihm ein frischer Trunk aufs Pferd gereicht. (Hoffmann, 1963c, S. 73)

Der Anblick des spannenden Augenblicks versetzt den Protagonisten Theodor in die Zeit seiner Lehrjahre, die er zum Teil in der Gesellschaft zweier italienischer Sängerinnen verbracht hat. Das imaginäre Bild am Anfang der Erzählung fungiert dabei als „Medium der Reflexion und Erinnerung“ (Krunić, 2002), das im Verlauf der Handlung ,zur Erschütterung und schließlich zur Selbsterkenntnis“ (ebd.) führt. Theodor erzählt dabei seinem Freund Eduard, dass er in den Gesichtszügen der dargestellten Sängerinnen 
die Schwestern Lauretta und Theresina, seine ehemaligen Betreuerinnen und Musen wiedererkennt. Die gemalten Frauenfiguren spiegeln die Charakterzüge der Künstlerinnen, wodurch die launische, temperamentvolle Lauretta und die stille, schüchterne Theresine „unterschiedliche Gesangstile und musikalische Repertoires innerhalb der italienischen Schule“ (Risi, 1998, S. 86) verkörpern.

Zugleich visualisiert die dargestellte Szene Theodors „Fermaten- und Erkenntnismoment.“ (Krunić, 2002) Das Fermaten-Motiv taucht mehrmals innerhalb der Erzählung auf und wird schließlich im Gemälde suggeriert, indem ,die Rundung der Laube dem Fermatenbogen [entspricht], der Reiter im Hintergrund dem fermatischen Punkt darunter“ (ebd.), wobei ,im Fortgang der Erzählung dann klar wird, dass der Reiter im Hintergrund des Bildes der eigentliche Mittelpunkt ist, die Hauptfigur Theodor nämlich.“ (ebd.) Somit erweist sich das Motiv als Verknüpfungspunkt zwischen Musik, Malerei und Literatur, da Hoffmann ,in seinem Text ein Bild in den Mittelpunkt [rückt], das seinerseits Musik darstellt.“ (Steinecke, 2004, S. 317) Diese Verbindung der Kunstarten prägt zugleich die Rolle des Hauptprotagonisten. „Theodor ist der Erzähler, er geht selbst (als Zuhörer und Zuschauer) in das Bild hinein, und er sieht in dem dirigierenden Abbate seine eigene frühere Rolle als Musiker widergespiegelt.“ (ebd.) Demnach verdeutlicht sich Hoffmanns Anspruch auf die romantische Universalpoesie auch in dieser Erzählung.

Als zweites Beispiel in dieser Reihe sei die Erzählung Doge und Dogaresse (1818) zu nennen, in der Hoffmann erneut ,eine Art Übersetzung und doch gravierende Umgestaltung eines bestimmten Werkes der bildenden Kunst in die Literatur“ (Klier, 1999, S. 29) gelingt. Diese Erzählung zählt zu Hoffmanns historischen Erzählungen, die um 1800 eine beliebte Gattung waren und deren Handlung meistens in das idealisierte Mittelalter versetzt wurde. Steinecke (ebd., S. 337) betont, dass sich Hoffmanns historische Erzählungen einerseits durch ,eine intensive Quellennutzung“ (ebd.) auszeichnen, „die bis in die wörtliche Übernahme ganzer Absätze reicht.“ (ebd.) $)^{2}$ Gleichzeitig bemüht sich Hoffmann, „die Freiräume der Fantasie [aufzubewahren - Anm. d. Verf.]“, sodass fantastische Elemente auch hier

\footnotetext{
${ }^{2}$ Als historische Quelle diente Johann Friedrichs Le Brets Staatsgeschichte der Republik Venedig (1796).
} 
vorhanden sind. Als Bildvorlage diente Carl Wilhelm Kolbes Gemälde Doge und Dogaresse, das 1816 in der Akademie der Künste zu Berlin ausgestellt wurde. Der Leser wird erneut durch die Gemäldeschilderung in die Handlung eingeführt, die sich in der Republik Venedig im 14. Jahrhundert abspielt.

Mit diesem Namen war in dem Katalog der Kunstwerke, die die Akademie der Künste zu Berlin im September 1816 ausstellte, ein Bild bezeichnet, daß der wackre, tüchtige C. Kolbe, Mitglied der Akademie, gemalt hatte und das mit besonderem Zauber jeden anzog, so daß der Platz davor selten leer blieb. Ein Doge in reichen prächtigen Kleidern schreitet, die ebenso reich geschmückte Dogaresse an der Seite, auf einer Balustrade hervor, er ein Greis mit grauem Bart, sonderbar gemischte Züge, die bald auf Kraft, bald auf Schwäche, bald auf Stolz und Übermut, bald auf Gutmütigkeit deuten, im braunroten Gesicht; sie ein junges Weib, sehnsüchtige Trauer, träumerisches Verlangen im Blick, in der ganzen Haltung. Hinter ihnen eine ältliche Frau und ein Mann, der einen aufgespannten Sonnenschirm hält. Seitwärts an der Balustrade stößt ein junger Mensch in ein muschelförmig gewundenes Horn, und vor derselben im Meer liegt eine reich verzierte, mit der venetianischen Flagge geschmückte Gondel, auf der zwei Ruderer befindlich. Im Hintergrunde breitet sich das mit hundert und aber hundert Segeln bedeckte Meer aus, und man erblickt die Türme und Paläste des prächtigen Venedig, das aus den Fluten emporsteigt. Links unterscheidet man San Marco, rechts mehr im Vorgrunde San Giorgio Maggiore. (Hoffmann, 1963c, S. 445)

Das Interesse der Museumsbesucher löst am Anfang der Erzählung eine Debatte aus. Die Teilnehmer versuchen zu klären, ob die Darstellung allegorisch ist, ein unglückliches Ehepaar präsentiert, oder eine geschichtlich nachweisbare Begebenheit. Bei der einleitenden Darstellung werden die geheimnisvollen und fantastischen Elemente durch den anlockenden, »besonderen Zauber« angedeutet, wodurch die „Unsicherheitsvermittlung und mehrdeutige[] Leseart des Gemäldes“ (ebd., S. 32) suggeriert wird. Die Antwort auf das Rätsel wird dabei durch die Erzählung eines Fremden entdeckt. Wie Kremer erklärt, fungiert das ausgestellte Bild innerhalb der Erzählung als „Scharnier zwischen den Fiktionsebenen“ (1998, S. 298), indem es erstmals innerhalb der Rahmenerzählung als imaginäres Artefakt geschildert wird, dessen Erklärung jedoch gleichzeitig zum Auslöser der Binnenerzählung wird. Melanie Klier (vgl. 1999, S. 37-39) betont, dass 
Hoffmanns Umgang mit dem Kolbe-Gemälde nicht einfach auf eine Vorlagenfunktion zurückzuführen sei, da die Bildkomposition einschließlich der Figurendarstellung innerhalb der Erzählung nicht eingehalten wird. Hoffmann dekonstruiert die Gemäldedarstellung, um dadurch die Erzählung zu konstruieren. Somit entsteht die unglückliche Liebesgeschichte zwischen dem Jüngling Antonio und der schönen Annunziata, der Gattin des alten Dogen Marino Falieri. Die im Erzählrahmen angesetzte „Kunstbetrachtung meint in diesem Sinn einen Prozess projektiver Rückkopplungen, in dem sich Fantasie und gemalte Darstellung wechselseitig beleben.“ (Kremer, 2009, S. 300) Die vorliegende Erzählung verdeutlicht Hoffmanns spielerischen Umgang mit der Bildvorlage, indem historische und fantastische Elemente in eine tragische Liebesgeschichte transformiert werden, die als Geheimnis der Gemäldedarstellung entlarvt wird.

Wie Deterding (ebd., S. 61) erklärt, setzt Hoffmann bei seinen Erzählungen, die ein Gemälde als Erzählanlass aufweisen, ein charakteristisches Verfahren ein, das sogenannte, ,ins Leben Treten[s] der geschaute[n] Gestalt.“ Durch dieses Darstellungsverfahren wird ,eine außen, in der Wirklichkeit, oder auch innen, nur in der Phantasie, gesehene und erkannte Gestalt in ein Werk (der Literatur) verwandelt- um dann, als dieses Werk, wieder „,ins Leben“ zu treten, nach außen: dem Leser gegenüber.“ (ebd.) Dabei sei ebenfalls zu betonen, dass die genannten Bilddarstellungen auf „der Abkehr von Mimesismodellen“ (ebd., S. 258) basieren. Das bedeutet, dass „Hoffmanns Bildvorstellungen auf die romantische Theorie der Imagination [zurückgreifen], die sich der Herstellung einer eigenen Wirklichkeit verschreibt und damit eine imaginäre Wirklichkeit der Kunst behauptet.“ (ebd., S. 358) Eine solche „Phantastik der Textproduktion“ (ebd.) lässt aufgrund der Bildvorlage neue Welten entstehen, die ihre Geheimnisse nur dem ,imaginär adäquaten Leser“" (ebd.) offenbaren.

Daher kann gesagt werden, dass bei den ausgewählten Erzählungen die einleitende Ekphrasis nicht nur als Erzählanlass fungiert. Da das im Erzählrahmen dargestellte Gemälde innerhalb der Binnenerzählung eine Umsetzung mit oder ohne Veränderungen erlebt, dient es ebenfalls als Verknüpfungselement zwischen den Fiktionsebenen. Die Gemäldeschilderung leitet die für die Erzählung charakteristischen Leitmotive ein oder erweist sich als eine Art Reflexionsmedium für die Protagonisten. Die 
Verbindung zwischen Literatur und Malerei bestimmt demnach sowohl den formalen als auch den inhaltlichen Aspekt dieser Werke.

\section{GESTALTUNG DER PROTAGONISTEN DURCH RÜCKGRIFFE AUF MALERISCHE VORBILDER}

Eine weitere Funktion, die der Malerei in Hoffmanns literarischem Werk zugeschrieben werden kann, bezieht sich auf die Gestaltung der Protagonisten. Risi (1998, S. 359) erwähnt, dass Hoffmanns Berufung auf malerische Vorbilder durch „punktuelle Referenzen auf spezifische Gemälde“ hergestellt wird, „die beispielsweise der Charakterisierung von Personen dienen.“ (ebd.) Folgende Beispiele präsentieren explizite Referenzen auf bestimmte Maler oder Gemälde, die Hoffmann bei der Gestaltung seiner Protagonisten einführt.

In diesem Zusammenhang wäre zunächst die Erzählung Abenteuer der Sylvester-Nacht (1815) zu erwähnen, in welcher der reisende Enthusiast während einer Silvesternacht in Berlin verschiedene Bekanntschaften schließt und dadurch in eine Welt an der Grenze zwischen Alltag, Traum und Fantasie versinkt. Der erste Teil der komplexen Erzählung schildert Begebenheiten aus dem Leben des reisenden Enthusiasten. Schmidt (vgl. 2006, S, 94-99) verweist darauf, dass Hoffmann alle Protagonisten außer dem reisenden Enthusiasten auf der Grundlage von Werken verschiedener Künstler wie Rembrandt, Mieris, Rubens, Callot oder sogar der zeitgenössisch populären Trugbilder (Phantasmagorien) gestaltet. Der vorliegende Überblick fokussiert sich jedoch nur auf die Frauengestalten und die Funktionen, welche die dabei erstellten Referenzen innerhalb der Erzählung einnehmen. Dabei muss hervorgehoben werden, dass ,es sich in dieser Erzählung nicht um reale Gestalten handelt, sondern um von Kunstwerken inspirierte Personifikationen von Aspekten des Ichs, sowie seiner Idealvorstellung, in deren Zusammenspiel das richtige oder falsche Verhältnis von Kunst und Leben diskutiert wird.“ (ebd., S. 95)

Der reisende Enthusiast begegnet im Weinrausch seiner ehemaligen Geliebten Julia, deren Erscheinung sich dem Verwirrten wie ,die Jungfrauen auf den Gemälden von Mieris“ (Hoffmann, 1963a, S. 377) offenbart. Schmidt identifiziert diesen Verweis auf Mieris mit zwei konkreten Gemälden und 
zwar mit „Die Liebesbotschaft und Die Musikstunde.“(Schmidt, 2006, 93) Betont wird, dass ,der Schnitt der Kleider um die Brust, Schultern, Nacken und Ärmel sowie das Haar der Frauen auf diesen beiden Gemälden, mit der Beschreibung in der Erzählung übereinstimmen." (ebd.) Außerdem wird darauf verwiesen, dass Hoffmann auch in „Rembrandts Gemälde Saskia mit der roten Blume“ (ebd.) nach den Zügen Julias suchte. Eine weitere geheimnisvolle Begegnung führt den reisenden Enthusiasten zu Erasmus Sphiker und seiner Geschichte vom verlorenen Spiegelbild. Die Gestalt der verführerischen Giulietta, durch die Sphiker sein Spiegelbild verlor, hat ebenfalls ihren Ursprung in den Gemälden mehrerer Künstler. Die aus dem „lichten Kerzenschimmer“ (Hoffmann, 1963a, S. 392) hervortretende Frauengestalt wird als ,ein Frauenbild von Rubens oder dem zierlichen Mieris“ (ebd.) beschrieben. „Das weiße Busen, Schultern und Nacken nur halb verhüllende Gewand, mit bauschigen, bis an die Ellbogen streifenden Ärmeln“ (ebd.) verweist auf den ,altertümlichen Putz“ (ebd.) der Frauenfiguren beider Meister. Schmidt (2006, S. 100) erklärt jedoch, dass diese Beschreibung ebenfalls auf Rubens verweisen könnte, indem „dieser Beschreibung wohl das stark mit Hell- Dunkel- Effekten arbeitende Gemälde von Judith am nächsten [kommt], die den Kopf des Holoferns in einen Sack steckt.“ Die Verbindung zu Rubens kann dabei nicht eindeutig hergestellt werden, da Hoffmann das Originalgemälde wahrscheinlich nicht gesehen hat, sondern nur zahlreiche Radierungen dieses Rubens-Motivs, die in Dresden ausgestellt waren. (vgl. ebd.) Der Verweis auf das Judith-Motiv bei Rubens markiert Hoffmanns Absicht, Giuliettas dunkle Seite zu enthüllen. Deren jungfräuliche Erscheinung wirft gleichzeitig einen tiefen Schatten, der sie in den Augen des Erasmus Sphiker als dämonische Verführerin erscheinen lässt.

Da zog es mich am Arm, der Kleine stand hinter mir mit dem alten Gesicht und lispelte: "Trink nicht, trink nicht - sieh sie doch recht an! - hast du sie nicht schon gesehen auf den Warnungstafeln von Breughel, von Callot oder von Rembrandt? "-Mir schauerte vor Julien, denn freilich war sie in ihrem faltenreichen Gewande mit den bauschigen Ärmeln, in ihrem Haarschmuck so anzusehen wie die von höllischen Untieren umgebenen lockenden Jungfrauen auf den Bildern jener Meister. (Hoffmann, 1963a, S. 389)

Wie Reher (vgl. 1997, S. 66 f.) betont, verzichtet Hoffmann auf die Beschreibung der Frau, indem er auf Breughel, Callot oder Rembrandt 
verweist. Somit spendet er dem gebildeten Leser eine bildliche Vorstellung, gleichzeitig auch einen emotionellen Gehalt, wodurch vor der anziehenden, jedoch verbotenen Verlockung des Frauenkörpers gewarnt wird. Die „dunkle“ (Hoffmann, 1963a, S. 387) Vision der entzückenden und verführerischen Julia, die dem Enthusiasten aus dem ,tiefsten Hintergrunde [des Spiegels]“ (ebd.) hervortrat, und später mit Giuliettas Erscheinung verschmelzen wird, weist deren Ursprung auch bei anderen Künstlern auf. Dazu kommt das Gemälde von Jan Brueghel dem Älteren „,Die Versuchung des heiligen Antonius (1603/1604)““ (Schmidt, 2006, S. 96), wo „die lichtumflossene Gestalt einer schönen jungen Frau dem heiligen Antonius [erschient]: monströse Fantasietiere im Dunkeln symbolisieren die verborgene Kehrseite der schönen Frau, nämlich die höllischen Strafen, die auf ihren Genuß folgen.“ (ebd.) Als weiteres Vorbild erweisen sich ebenfalls Callots Radierungen genauer die Darstellungen der Todessünden „Superbia, Pigritia und Gula (1618-1625).“ (ebd.) Jede der Todessünden ,„[wird- Anm. d. Verf.] jeweils mit einem Tier, das die Sünde des Hochmuts, der Trägheit und Völlerei verkörpert, [dargestellt] sowie mit einem oder mehreren drachengeflügelten Teufelchen begleitet - die die von der Frau und dem Tier verkörperte Sünde bestrafen.“ (ebd.) Hoffmann entdeckt in den dramatischen Kompositionen barocker Künstler ein Frauenbild, das zugleich als sanfte Jungfrau und dämonische Verführerin erscheint.

Schmidt betont dabei, dass durch die Frauendarstellungen „Hoffmann auf [die- Anm. d. Verf.] christlichen Warnungen vor unsublimiertem Genuss [zurückgreift], um zwischen der Liebe zur Kunst und der Liebe zu einer konkreten Frau, zwischen Transzendentem und Konkretem, Ideal und Wirklichkeit zu differenzieren.“ (ebd., S. 106) Die genannten Opposition bilden dabei den thematischen Kern der Erzählung. Durch Verweise auf verschiedene Frauendarstellungen der barocken Malerei setzt sich Hofmann zugleich auch mit der ,romantische[n] Kunstvorstellung von der auf das Transzendente, auf das Ideale gerichteten Kreativität des Künstlers“ (ebd., S. 107) auseinander. Seine Position erweist sich somit als „entscheidende[] Verlagerung gegenüber Schlüsseltexten der Romantik“ (ebd.) und ,[macht] die Unterscheidung zwischen Ideal und Wirklichkeit dem Künstler [zur Aufgabe], statt sie [...] zur Deckung zu bringen.“ (ebd.)

Auch bei der Darstellung Cyprians und des wahnsinnigen Grafen P** am Beginn der Erzählung Der Einsiedler Serapion (1819) greift Hoffmann 
auf eine entsprechende malerische Vorlage. Das wunderliche Zusammentreffen erscheint dem Erzähler wie eine Vergegenwärtigung des biblischen Motivs der Begegnung des Heiligen Antonius und Paulus. Die Erscheinung des „wahnsinnigen Anachoreten“ (Hoffmann, 1963c, S. 22) mit „langem schwarzen verwildertem Bart“ (ebd.) in „brauner Einsiedlerkutte“ (ebd.) trägt die Züge des ikonografischen Motivs, das „man nur auf Bildern sah oder nur aus Büchern kannte.“" (ebd.) Bekannte Darstellungen dieses Motivs findet man innerhalb der italienischen Malerei der Renaissance z. B. bei Pier Francesco Sacchi (1485-1528), Giovanni Girolamo Savoldo (um 1480-1548) oder der altdeutschen bei Mathias Grünewald (um 1475/1480-1528) und Albrecht Dürer (1471-1528). Hoffmanns Figuren werden jedoch in eine von Menschen abgesonderte „Bergschlucht auf einem Felsstück“ (ebd.) versetzt, die Ähnlichkeiten mit Salvator Rosas Landschaft mit dem heiligen Antonius Abbas und Paulus dem Eremit (1660 - 1663) aufweist. Dabei ist wichtig zu erwähnen, dass Salvator Rosa als einer der wichtigsten Vertreter der malerischen Nachtstück-Gattung gilt, deren Ästhetik man auch innerhalb der Erzählsammlung Die Serapionsbrüder wiederbegegnet. Katrin Bomhoff (vgl. 1999, S. 130) erklärt, dass sich eine derartige Umsetzung des Motivs mit einem wahnsinnigen, zugleich aber meditierenden Einsiedler in einer durch Rosa inspirierten Felsenlandschaft als Besonderheit von Hoffmanns Darstellung erweist. So entsteht ein Kontrast zwischen der statischen Figur und der dynamischen Landschaft. „Eben diese Kontraste sind für Hoffmann von außerordentlicher Bedeutung, denn sie stellen ein Gestaltungsmittel dar, daß sich als bestimmend für das Kunstschaffen beider, des Dichters und des bildenden Künstlers, herausstellen wird.“ (ebd.) Hoffmanns Anliegen besteht in der Identifikation der eigenen Poetik mit Rosas Ästhetik, indem er durch das Anfangsbild eine Art „,vorweggenommene Programmatik“ (ebd., S. 131) der Erzählsammlung vorlegt. Das Düstre, Wilde und Geheimnisvolle, das sich als Besonderheit von Rosas Stil erweist, soll daher auch die Erzählungen der Sammlung prägen. ,[Hoffmann-- Anm. d. Verf.] bietet dem Leser diesmal keine Vorrede zur Kennzeichnung seiner Dichtung nach der »Manier« eines bildenden Künstlers, sondern führt ihm in noch verkürzter Form seine Absichten vor die Augen.“ (ebd.) Die Konzeption der Anfangsszene soll demnach „,bereits alle für den Zyklus relevanten Motive und Erkenntnisse“" (ebd.) suggerieren. 
Hoffmann greift bei der Darstellung seiner Protagonisten auf malerische Vorbilder zurück, die ebenfalls den Gattungen der Fantasie- und Nachtstücke entstammen. Dies bezieht sich sowohl auf die Motive ausgewählter malerischer Vorlagen als auch auf die Licht-Schatten Effekte, durch die Hoffmann die Erscheinungen seiner Protagonisten betonen möchte. Dabei ist es wichtig zu erwähnen, dass gerade durch die Verweise auf bestimmte Künstler Hoffmann einfache Beschreibungen vermeiden möchte. Klier (1999, S. 38) betont dabei, dass sich Hoffmann besonders bei Frauendarstellungen von der Einstellung leiten ließ, „,[dass- Anm. d. Verf.] [Schönheit] über das Medium der Sprache nicht treffend zu visualisieren ist.“ Deshalb greift er auf ein Syntheseverfahren, eine „literarische[] Portraitkonstruktion“ (ebd.), die sich als „eine bewußt von Autor inszenierte und evozierte Andeutungskunst" (ebd.) entlarvt. Durch den Verweis auf das entsprechende malerische Gegenstück wird „der Leser gezwungen, seine individuelle Weltkenntnis ins Spiel zu bringen, um das von Hoffmann imaginierte Frauenbild entstehen zu lassen.“ (ebd.) Eine Andeutung erweist sich dabei als ausreichend, da sie einerseits dem Leser einen Vorstellungsrahmen spendet, gleichzeitig aber genug Freiraum für die spielerische Entfaltung seiner Fantasie freilässt.

\section{GESTALTUNG LITERARISCHER LANDSCHAFTEN DURCH RÜCKGRIFFE AUF MALERISCHE VORBILDER}

Hoffmanns literarische Landschaftsdarstellungen weisen ebenfalls eine Verbindung zu seinen malerischen Vorbildern auf, wodurch im vorliegenden Überblick die letzte Funktion anhand zweier Beispiele markiert wird. In diesem Zusammenhang sei erneut Salvator Rosa zu nennen. Verschiedene Bezüge zum Leben und Werk des italienischen Landschaftsmalers lassen sich besonders innerhalb der Sammlung Die Serpionsbrüder erkennen. Rosas Malerei charakterisiert die Dynamik und Dunkelheit seiner „düsteren und bizarren“ (Kremer, 1998, S. 156) Landschaften, in die Hoffmann die fantastischen Begebenheiten seiner Erzählungen verortet. Die Anfangsszene der Erzählung Der Einsiedler Serapion referiert explizit darauf, indem die Begegnung der Protagonisten in „Salvator Rosas wilde[s] Gebirge“ (Hoffmann, 1963c, S. 22) versetzt wird. Hoffmann gestaltet eine ungemütliche Umgebung mit düsteren, vorüberziehenden Wolken und wenig Licht. Die mystische und schaurige Stimmung dieser Landschaft 
erfüllt somit die Rahmenerzählung und lässt die Begegnung zwischen Cyprian und dem Einsiedler noch ungewöhnlicher und dramatischer Erscheinen. Wie Bomhoff (1999, S. 132) erklärt ,gibt [Rosa - Anm. d. Verf.], wie viele Landschaftsmaler, eine in dem Sinne konstituierte und idealisierte Natur wieder, als sie sich aus verschiedenen Versatzstücken zusammensetzt und nicht dem Vorgefundenen treu abgebildet ist.“ In Bezug auf Rosas Vorstellungen wird auch Hoffmanns Verhältnis zur Landschaft diesem Idealisierungsanspruch unterworfen.

„Hoffmann schätzt dieses Verfahren, das die gegebene Wirklichkeit der eigenen Anschauung unterordnet, ohne sie zu missachten. Die Nachahmung des Vorgefundenen kann es bei ihm nicht geben ohne die Forderung nach dem Entwurf des unverkennbar Eigenen in der wiederum verlebendigenden Verwandlung der Kunst.“ (ebd.)

Hoffmanns Landschaften gehören daher ebenfalls einer fantastischen Welt des „Fremdartigen“ (Hoffmann, 1963c, S. 22) und „Seltsamen“ (ebd.) jenseits der gegebenen Wirklichkeit an, was durch den Verweis auf Rosa zusätzlich betont wird. So werden auch die Landschafsdarstellung der Ästhetik des Fantastischen und Schauerlichen angepasst. Wie Bomhoff (1999, S. 135) betont, ,[handelt] Rosa den Kompositionsmustern der Landschaftsmalerei des 17. Jahrhunderts gänzlich zuwider, wenn er die Staffagefiguren seiner Bilder mit ihrer jeweiligen Umgebung kontrastiert.“ Ähnliches lässt sich ebenfalls bei Hoffmann bemerken, der „,[in seiner Erzählung] ebenso wenig [...] die Gestalt in Einklang mit dem Charakter der umgebenden Landschaft [bringt]. (ebd.) Dabei sei zu betonen, dass Hoffmanns Werke keine ausführliche Auseinandersetzung mit der Natur aufweisen. „Natur findet sich eher als Kulisse und Metapher für das Erzählte denn als in den Mittelpunkt gerückter Gegenstand der Erörterung wieder.“ (ebd., S. 134) Die abgesonderte „Bergschlucht“ (ebd.), in die die Erzählung verortet wurde, fungiert hier als ,theatergleiche[] Szenarie“" (Bomhoff, 1999, S. 132) für die Rahmenbegegnung, die sowohl den Erzähler als auch den Leser einen „leisen Schauer“ (Hoffmann, 1963c, S. 22) spüren lässt. Die imaginäre Landschaft dient somit der Vermittlung der geheimnisvollen und düsteren Stimmung, indem sie die schauerliche, zugleich aber anziehende Seite der Natur entdeckt. 
In diesem Zusammenhang wäre ebenfalls die Erzählung Die Jesuitenkirche in G. (1816) zu nennen. Die Bezeichnung der Erzählung ,als programmatisches »Nachtstück«" (Schneider, 2015, S. 56) lässt sich besonders durch verschiedene Bezüge auf Rosa erklären. Wie Schneider betont, „wird mit den im malerischen Hell-Dunkel gehaltenen nächtlichen Malszenen und dem Verweis auf die Schauder erregende Bildästhetik der Landschaften Salvator Rosas [...] ein expliziter Bezug zur intermedialen Herkunft des Begriffs aus der Malerei gegeben.“ (ebd.) Dies bestätigen einzelne Szenen, die in eine schaurige und bedrohliche Landschaft versetzt wurden. Als solche erweist sich Bertholds Waldspaziergang in der Umgebung Roms. Dabei erscheint dem verzweifelnd nach künstlerischer Inspiration suchenden Maler die Natur als „bedrohliches Ungeheuer“ (Hoffmann, 1963b, S. 497), das „Untergang und Verderben“ (ebd.) ankündigt. Das „unheimliche[] Grauen“ (Hoffmann, 1963b, S. 497) des „dunklen Wald[es]“ (ebd.), hinter dem „ferne[] Berge“ (ebd.) emporragen, weist daher seinen Ursprung in Rosas zahlreichen Landschaftsbildern auf, die um 1800 vor allem durch Kupferstichkopien in Deutschland Verbreitung fanden. Es muss ebenfalls betont werden, dass neben der Landschaftsdarstellung die Erzählung einen weiteren Bezug zu Rosa herstellt. Schmidt erkennt diese Verbindung im thematischen Kern der Erzählung. Während der Maler Berthold sein künstlerisches Ideal nach dem Vorbild Raffaels sucht, trägt sein tragisches Schicksal die geheimnisvollen und dunklen Züge der Künstlerfigur Rosas. Es handelt sich daher um die Kluft zwischen „,er Erhabenheit des alles gelobten, an Raphael orientierten fiktiven Werks des Protagonisten einerseits und andererseits des Unheimlichen, Bedrohlichen und Spannungsreichen des eher an Salvator Rosa gemahnenden Stils der Erzählung." (Schmidt, 2006, S. 134)

Wie bereits erwähnt wurde, gilt Salvator Rosa als eines der wichtigsten künstlerischen Vorbilder Hoffmanns. Die Schauerästhetik des Landschaftsmalers prägt nicht nur die Erzählungen der Nachtstücke, sondern bestimmt ebenfalls den programmatischen Aspekt der späteren Sammlung Die Serapionsbrüder. Eine Ausprägung erlebt dieser Einfluss bei der Gestaltung literarischer Landschaften. Hoffmans Landschaftsszenen erweisen sich daher ebenfalls als literarische Nachtstücke nach Rosas Vorbild. Deren kulissenartige Funktion soll dabei die Schauerstimmung der jeweiligen Szene oder der ganzen Erzählung betonen. 


\section{SCHLUSSBEMERKUNG}

Eine Trennung der Künste war für E. T. A. Hoffmann unbekannt, vielmehr sah er den wahren Ausdruck erst in deren Verbindung. Die Vorliebe zur Malerei, die während seiner künstlerischen Entwicklung eine sekundäre Rolle einnehmen musste, erwies sich jedoch als entscheidend für die Bestimmung und Begründung seiner Erzählprogrammatik. Abschließend kann gesagt werden, dass die in diesem Text präsentierten Verweise auf diverse Maler oder konkrete Gemälde innerhalb der Erzählungen verschiedenen Funktionen unterliegen. Als intermediale Verknüpfungen fungieren sie als poetologischer Selbstkommentar und verweisen somit auf Hoffmanns Umsetzung des romantischen Konzepts der Universalpoesie. Die Verweise deuten dabei ebenfalls auf seine Auseinandersetzung mit der frühromantischen Ästhetik oder dienen der Behandlung und Betonung verschiedener künstlerischer Problemfragen. All dies kann aufgrund der Literarisierung fantastischer Verwandlungen und Vorgänge, die Darstellung der Protagonisten oder landschaftlicher Szenarien verfolgt werden. Hoffmanns, schon bei Zeitgenossen beliebte Fantastik- und Schauerpoetik zeichnet sich durch eine intermediale Vielseitigkeit und Komplexität aus, die seine literaturgeschichtliche Rezeption als einen der beliebtesten Autoren der deutschen Romantik unterstreicht.

\section{LITERATURVERZEICHNIS}

Bomhoff, K., 1999. Bildende Kunst und Dichtung. Die Selbstinterpretation E. T. A. Hoffmanns in der Kunst von Jacques Callots und Salvator Rosas. Freiburg: Rombach Verlag.

Brandl-Risi, B., 2015. Bild/Gemälde/Zeichnung. In: Lubkoll, C., Neumeyer, H. Hg. E. T. A. Hoffmann Handbuch. Stuttgart: J. B. Metzler.

Braun, P., 2004. E. T. A. Hoffmann. Dichter, Zeichner, Musiker. Düsseldorf/Zürich: Artemis\& Winkler.

Deterding, K., 2009. E. T. A. Hoffman. Erzählungen und Romane. Einführung in Leben und Werk, 2. Band 3. Würzburg: Königshausen \&Neumann.

Göbel, H., 1992. E. T. A. Hoffmann als Maler und Zeichner. In: Arnold, H. L. Hg. E. T. A. Hoffmann. Text und Kritik. München: Weber Offset GmbH.

Hoffmann, E. T. A., 1963. Poetische Werke in sechs Bänden. Band 1. Berlin: Aufbau Verlag. 
Hoffmann, E. T. A., 1963. Poetische Werke in sechs Bänden. Band 2. Berlin: Aufbau Verlag.

Hoffmann, E. T. A., 1963. Poetische Werke in sechs Bänden. Band 3. Berlin: Aufbau Verlag.

Kaiser, G. R., 1988. E. T. A. Hoffmann. Stuttgart/Weimer: J. B. Metzler.

Klier, M., 1999. Kunstsehen. E. T. A. Hoffmanns literarisches Gemälde Doge und Dogaresse. In: Steinecke, H. u. a. Hg. E. T. A. Hoffmann Jahrbuch. Mitteilungen der E.T.A. Hoffmann-Gesellschaft. Berlin: Erich Schmidt Verlag.

Knöferl, E., 2015. Ritter Gluck. Eine Erinnerung aus dem Jahre 1809 (1809). In: Lubkoll, C., Neumeyer, H. Hg. E. T. A. Hoffmann Handbuch. Stuttgart: J. B. Metzler.

Kremer, D., 1998. E. T. A. Hoffmann zur Einführung. Hamburg: Junius. (Hg.)

Kremer, D., 2009. De Gruyter Lexikon. E. T. A. Hoffmann. Leben-Werk-Wirkung. Berlin/New York: De Gruyter.

Kremer, D., Klicher, A. B., 2015. Romantik. Stuttgart/Weimar: J.B. Metzler.

Krunić, D., 2002. Integration von Elementen der Bildenden Kunst und der Musik dargestellt an E. T. A. Hoffmanns „Die Fermate.“ [online]. München: Goethezeitportal e.V. Gesellschaft zur wissenschaftlichen Förderung und kulturellen Vermittlung von Literatur, Kunst und Kultur der Goethezeit auf medialer Basis (VR18002). Verfügbar unter: http://www.goethezeitportal.de/ wissen/projektepool/intermedialitaet/autoren/etahoffmann/diefermate/hummel.html (06. 11. 2002) [letzter Zugang am 17. 09. 2020].

Ponert, J. D., 2012. E. T. A. Hoffmann-Das Bildkünstlerische Werk. Ein kritisches Gesamtverzeichnis. Band 2: Abbildungen. Bamberg: Michael Imhof Verlag.

Ponert, J. D., 2012. E. T. A. Hoffmann-Das Bildkünstlerische Werk. Ein kritisches Gesamtverzeichnis. Band 1: Text. Bamberg: Michael Imhof Verlag.

Reher, S., 1997. Leuchtende Finsternis. Erzählen in Callots Manier. Köln/ Weimar/ Wien: Böhlau Verlag.

Safranski, R., 1984. E. T. A. Hoffmann. Das Leben eines skeptischen Phantasten. München/Wien: Carl Hanser Verlag.

Schanze, H., 2018. Erfindung der Romantik. Stuttgart: Metzler.

Schneider, S., 2015. Die Jesuitenkriche in G. (1816). In: Lubkoll, C., Neumeyer, H. Hg. E. T. A. Hoffmann Handbuch. Stuttgart: J. B. Metzler.

Schmaus, M., 2015. Der goldene Topf. Ein Märchen aus der neuen Zeit (1814). In: Lubkoll, C., Neumeyer, H. Hg. E. T. A. Hoffmann Handbuch. Stuttgart: J. B. Metzler. 
Schmidt, R., 2006. Wenn mehrere Künste im Spiel sind. Intermedialität bei E. T. A. Hoffmann. Göttingen: Vandenhocek \& Ruprecht.

Schnyder, P., 2015. Jacques Callot (1814). In: Lubkoll, C., Neumeyer, H. Hg. 2015. E. T. A. Hoffmann Handbuch. Stuttgart: J. B. Metzler.

Steinecke, H., 2004. Die Kunst der Fantasie. E. T. A Hoffmanns Leben und Werk. Frankfurt am Main: Insel Verlag.

Wittkop-Ménardeu, G., 1996. E. T. A. Hoffmann. Hamburg: Rowohlt. 


\title{
FUNKCIJE LIKOVNE UMJETNOSTI U KNJIŽEVNOM DJELU E. T. A. HOFFMANNA
}

\begin{abstract}
Sažetak
E. T. A. Hoffmann je bio svestran umjetnik koji se prvenstveno proslavio kao književnik, ali i kao kompozitor i karikaturista. Hoffmanova sklonost ka muzici i likovnoj umjetnosti oslikava se također u njegovom književnom opusu. Od rane faze pa sve do kasnih ostvarenja Hoffmann u svojim djelima, prvenstveno u pripovijetkama, stvara različite poveznice između likovne umjetnosti i književnosti. Pri tome eksplicitno upućuje na određene umjetnike ili djela s kojima se susretao tijekom boravaka u Dresdenu, Berlinu i Bambergu. Među Hoffmannovim likovnim uzorima posebno se izdvajaju fancuski barokni grafičar Jacques Callot (1592-1635) i talijanski slikar pejzaža Salvator Rosa (1615-1673), ali brojne reference upućuju i na slikare kao što su Hieronymus Bosch ili Pieter Brueghel stariji, Rubens, Rembrandt ili Dürer. U radu se nastoje predstaviti različite funkcije navedenih referenci unutar odabranih pripovjedaka iz zbirki Fantasiestücke in Callots Manier. Blätter aus dem Tagebuche eines reisenden Enthusiasten (1814/15), Nachtstücke (1816/17), te Die Serapionsbrüder (1819/21). Predstavljeni osvrt u kontekstu intermedijalne književne teorije nastoji doprinijeti boljem razumijevanju kako poetološkog tako i sadržajnog aspekta Hoffmannovih djela.
\end{abstract}

Ključne riječi: E. T. A. Hoffmann, romantizam, intermedijalnost, književnost, slikarstvo 


\title{
THE FUNCTIONS OF FINE ARTS IN THE LITERARY WORK OF E. T. A. HOFFMANN
}

\begin{abstract}
Summary
E. T. A. Hoffmann was a multi-talented artist who was not only known as a writer but as well as a composer and a draftsman. These talents can be noticed through various traces within his literary work. The references to the fine arts characterize Hoffmann's entire oeuvre from the early to the late phase. The interest in certain artists and works, which Hoffmann adopted both in Dresden and during the Bamberg and Berlin periods, can be encountered through various references, especially within his narratives. The presented article includes three story collections: Fantasiestücke in Callots Manier,. Blätter aus dem Tagebuche eines reisenden Enthusiasten (1814/15), Nachtstücke (1816/17), and Die Serapionsbrüder (1819/21). It begins by referring to Hoffmann's engagement with various artistic models, and then goes on to examine the traces of these encounters. The approach divides the references into four categories that within selected narratives reference to different features. Hoffmann not only relays on the traditionally painterly genres of fantasy and nocturne, but also attempts to transpose their characteristics into literature. The works of visual artists can serve as narrative prompts, or to shape the protagonist and landscape depictions. The above-mentioned references contribute to a better understanding of Hoffmann's narrative work, both on the poetological level and on the level of content.
\end{abstract}

Key words: E. T. A. Hoffmann, Romanticism, intermediality, literature, painting 